\title{
The challenges of miniaturization solar plane
}

\author{
Mohammad VAEZI \\ Department of Mechanical Engineering, Najafabad Branch, Islamic Azad University, \\ Najafabad, Iran.
}

\begin{abstract}
In many aerial vehicles applications, the flight duration is a key factor for the success of a mission. A solution to improve significantly this endurance is the use of solar energy. This chapter presents a conceptual design methodology for the sizing of solar-powered airplanes, applicable to MAVs as well as to manned sailplanes, that optimizes the sizing of the different elements. It uses mathematical models, for weight prediction for example, that were studied on a very large range. This allows to clearly point out the problems that occur when scaling down solar MAVs.
\end{abstract}

Keywords: Air transport, Endurance, Solar aircraft, Solar energy.

\section{Introduction}

This chapter aims at investigating precisely the problems that occur when targeting solar flight at the MAV size, by focusing on the global design of such flying platforms. We will not tackle the precise problem of airfoil selection only, or propeller optimization, but rather concentrate on the optimal sizing of all the elements and particularly emphasize the scaling effects. For this purpose, we will introduce the design methodology that was developed within the framework of the Rad-Solar plane project1 at the Autonomous Systems Lab. Then, using the mathematical models developed for each subpart, such as the mass and efficiency of a motor with respect to its power, the advantages and drawbacks of downscaling will be presented. That will allow an adaptation of the parameters used in the methodology in order to design a solar MAV.

\section{Design Methodology}

The methodology that we propose is based on two simple balances: 
- Weight balance: the lift force has to be equal to the weight of all the elements constituting the airplane.

- Energy balance: the energy that is collected during a day or the light period from the solar panels has to be equal or higher than the electrical energy needed by the motor during the entire flight. These two balances are represented in Fig.1 where it is clear that the propulsion group and the airframe cannot be dimensioned without knowing the total mass to lift, but this value is the sum of the masses of all the airplane elements that will be sized according to the energy required by the propulsion group. From here on, and considering the type of mission and the payload to embed, there could be two different methods to solve this loop, i.e., to design the airplane.

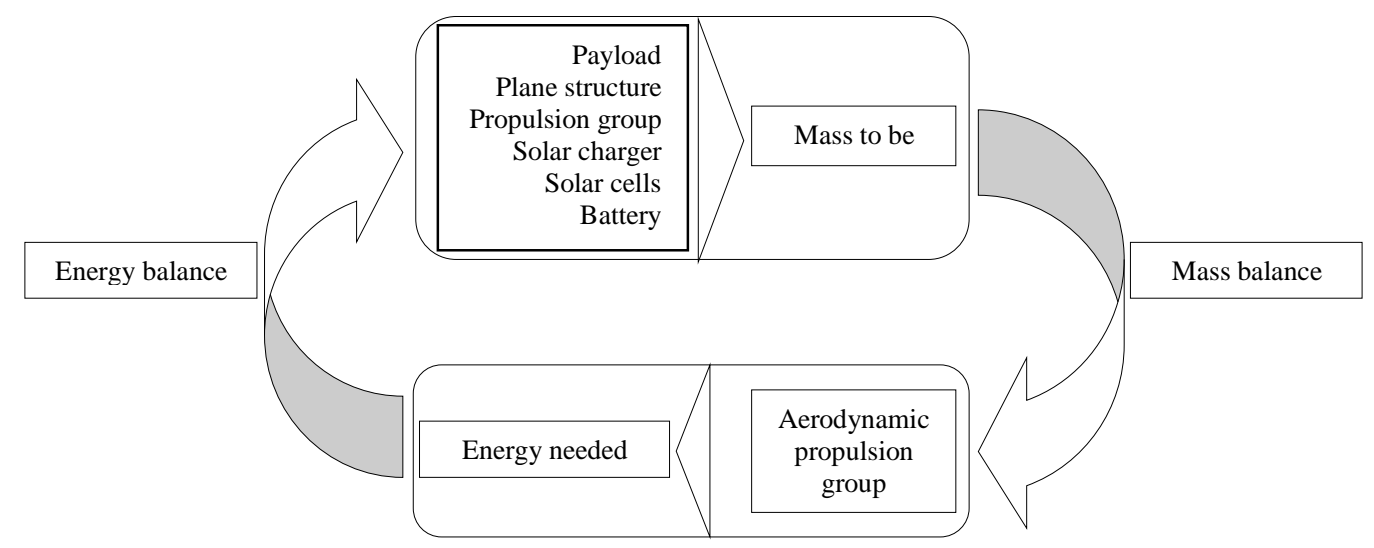

Fig. 1: Energy and mass balance

- The discrete and iterative approach would consist in selecting a first set of components (motor, solar panels, battery, etc.) based on pure estimation of the final required power or on previous designs. Then, having their total mass, the wing surface and propulsion group could be sized. Having chosen a precise motor, gearbox, and propeller, one could calculate the power needed for level flight. This value would then be compared with the power available from the previously selected solar generator, and so on, an iterative process would take place with at each time refining selection, improving the design, ending hopefully with a converging solution.

- The approach used here is an analytical and continuous approach that consists in describing all the relations between the components with analytical equations using models describing the characteristics of each of them. This method has the benefit of directly providing a unique and optimized design, but requires very good mathematical models. In the present case, an important effort was made to make these 
models accurate on a very wide range, so that the methodology could be applied to solar MAVs as well as to manned solar airplanes. The first steps are thus to establish the expression of the power needed for the aircraft in level flight, the solar energy available, and then develop the weight prediction models for all the airplane elements. All the details of these developments are summarized in compact form in Fig.2. It represents the problem of solar airplane conceptual design in a graphical approach and in fact contains the same loop that is depicted in Fig.1 but displayed in a compact mathematical manner.

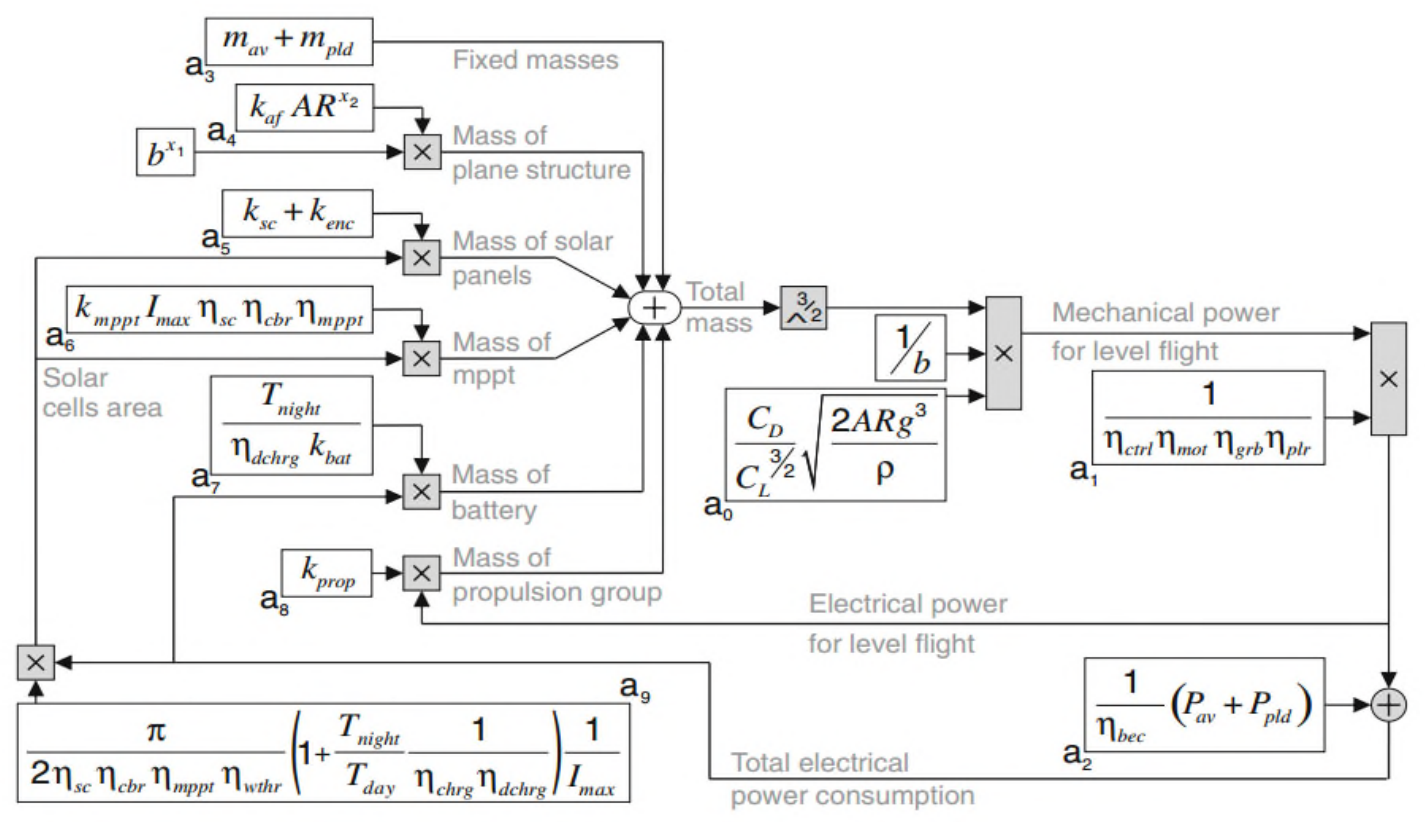

Fig. 2: Schematic representation of the design methodology.

- In order to extract meaningful information, it is necessary, among the 30 parameters that our model contains, to distinguish between three different classes: The first group is composed of the parameters which are linked to a technology and are constant or can be regarded as constant for very good design. This is for example the case of motor or propeller efficiencies that should be around $85 \%$ when optimized for a specific application.

- The second group of parameters is linked to the mission: they are the air density, given by the flight altitude; the day and night duration, depending on the time and the location; and the mass and power consumption of the payload. 
- $\quad$ Finally, the last group is composed of the parameters that we vary during the optimization process in order to determine the airplane layout, that is why we should use here the term variable rather than parameter. They are the wingspan and the aspect ratio of the wing.

A complete listing of these parameters is presented in Tables.1, 2, and 3. The values that are mentioned were used for the design of the $3.2 \mathrm{~m}$ wingspan Rad-Solar plane prototype. The process to solve the loop analytically is quite simple. Considering the point where the masses of all elements are summed up in Fig.2 and using the substitution variables $\mathrm{a}_{\mathrm{i}}$, we can write:

$$
\begin{aligned}
& m-\underbrace{a_{0} a_{1}\left(a_{7}+a_{8}+a_{9}\left(a_{5}+a_{6}\right)\right)}_{a_{10}} \frac{1}{b} m^{\frac{3}{2}}=\underbrace{a_{2}\left(a_{7}+a_{9}\left(a_{5}+a_{6}\right)\right)+a_{3}}_{a_{11}}+a_{4} b^{x_{1}} \\
& m-\underbrace{a_{10} \frac{1}{b}}_{a_{12}} m^{\frac{3}{2}}=\underbrace{a_{11}+a_{4} b^{x_{1}}}_{a_{13}}
\end{aligned}
$$

Or more compact

$$
m-a_{12} m^{\frac{3}{2}}=a_{13}
$$

Equation has only a positive non-complex solution, which makes physical sense, if

$$
a_{12}^{2} a_{13} \leq \frac{4}{27}
$$

The conceptual design process can thus be summarized as follows: after having set the mission requirements and chosen the technological parameters, one can try many possible airplane layouts by changing b and AR. The condition on Eq. (4) tells directly

Table 1: Parameters that are constant or assumed constant

\begin{tabular}{llll}
\hline Parameter & Value & Unit & Description \\
\hline $\mathrm{C}_{\mathrm{L}}$ & 0.8 & - & Airfoil lift coefficient \\
$\mathrm{C}_{\mathrm{D} \text { alf }}$ & 0.013 & - & Airfoil drag coefficient \\
$\mathrm{C}_{\mathrm{D} \text { par }}$ & 0.006 & - & Parasitic drag coefficient \\
$\mathrm{e}$ & 0.9 & - & Oswald's efficiency factor \\
$\mathrm{I}_{\max }$ & 950 & {$\left[\mathrm{~W} / \mathrm{m}^{2}\right]$} & Maximum irradiance \\
$\mathrm{K}_{\mathrm{bat}}$ & 190.3600 & {$[\mathrm{~J} / \mathrm{Kg}]$} & Energy density of Li-Ion \\
$\mathrm{K}_{\mathrm{sc}}$ & 0.32 & {$\left[\mathrm{~kg} / \mathrm{m}^{2}\right]$} & Mass density of solar cells \\
$\mathrm{K}_{\mathrm{enc}}$ & 0.26 & {$[\mathrm{~kg} / \mathrm{w}]$} & Mass density of encapsulation
\end{tabular}


Bulletin de la Société Royale des Sciences de Liège, Vol. 85, 2016, p. 863 - 878

\begin{tabular}{llll}
$\mathrm{K}_{\mathrm{mppt}}$ & 0.00042 & {$[\mathrm{~kg} / \mathrm{w}]$} & Mass to power ratio of MPPT \\
$\mathrm{K}_{\mathrm{prop}}$ & 0.0008 & {$\left[\mathrm{~kg} / \mathrm{m}^{3}\right]$} & Mass to power ratio of prop. group \\
$\mathrm{K}_{\mathrm{af}}$ & $0.44 / 9.81$ & {$[\mathrm{~kg}]$} & $\begin{array}{l}\text { Structural mass constant } \\
\mathrm{m}_{\mathrm{av}}\end{array}$ \\
$\eta_{\mathrm{bec}}$ & 0.15 & - & Mass of autopilot system \\
$\eta_{\mathrm{cs}}$ & 0.65 & - & Efficiency of step-down converter \\
$\eta_{\mathrm{cbr}}$ & 0.90 & - & Efficiency of solar cells \\
$\eta_{\mathrm{chrg}}$ & 0.95 & - & Efficiency of the curved solar panels \\
$\eta_{\mathrm{ctrl}}$ & 0.95 & - & Efficiency of battery charge \\
$\eta_{\mathrm{dchrg}}$ & 0.95 & - & Efficiency of motor controller \\
$\eta_{\mathrm{grb}}$ & 0.97 & - & Efficiency of battery discharge \\
$\eta_{\mathrm{mot}}$ & 0.85 & - & Efficiency of gearbox \\
$\eta_{\mathrm{mppt}}$ & 0.97 & - & Efficiency of motor \\
$\eta_{\mathrm{plr}}$ & 0.85 & - & Efficiency of MPPT \\
$\mathrm{P}_{\mathrm{av}}$ & 1.5 & {$[\mathrm{~W}]$} & Efficiency of propeller \\
$\mathrm{X}_{1}$ & 3.1 & - & Power of autopilot system \\
$\mathrm{X}_{2}$ & -0.25 & - & Airframe mass area exponent \\
\hline
\end{tabular}

Table 2: Parameters determined by the mission

\begin{tabular}{llll}
\hline Parameter & Value & Unit & Description \\
\hline $\mathrm{M}_{\text {pld }}$ & 0.05 & {$[\mathrm{~kg}]$} & Payload mass \\
$\eta_{\text {whtr }}$ & 0.7 & - & Irradiance margin factor \\
$\mathrm{P}_{\text {pld }}$ & 0.5 & {$[\mathrm{~W}]$} & Payload power consumption \\
$\rho$ & 1.1655 & {$\left[\mathrm{~kg} / \mathrm{m}^{3}\right]$} & Air density $(500 \mathrm{~m})$ \\
$\mathrm{T}_{\text {day }}$ & 13.2 .3600 & {$[\mathrm{~s}]$} & Day duration \\
\hline
\end{tabular}

if the design is feasible or not with this wingspan and aspect ratio. In the case of a positive answer, the total mass $\mathrm{m}$ can be found, which will constitute the starting point for the calculation of the power and characteristics of all the other elements. Hence, this method is not aimed at being used to optimize a precise and local element like the airfoil or the propeller, its objective is rather to help to choose the best combination and size of the different elements. 
Table 3: Variables linked to the airplane shape

\begin{tabular}{llll}
\hline Parameter & Value & Unit & Description \\
\hline AR & 12.9 & - & Aspect ratio \\
$\mathrm{b}$ & 3.2 & {$[\mathrm{~m}]$} & Wingspan \\
$\mathrm{m}$ & 2.5 & {$[\mathrm{~kg}]$} & Total mass \\
\hline
\end{tabular}

\section{Methodology Application}

In order to see how it can be concretely applied, we will present here the example of the airplane. The objective here is to design an UAV that can embed a small payload of around $50 \mathrm{~g}$, but that can achieve continuous flight at constant altitude over $24 \mathrm{~h}$ using only solar energy. The mission and technological parameters that were used are presented in Tables 1, 2. Using these parameters and trying various airplane shapes, i.e., wingspan from 0 to $6 \mathrm{~m}$ and different aspect ratios, Eq. (4) determines if the solution is feasible, in which case Eq. (3) is solved to find the airplane gross mass (Fig. 3). Having found for each possibility the total mass, one can then introduce it in the loop of Fig.2 to calculate precisely all the other airplane characteristics:

powers at propeller, gearbox, motor and battery, surface of wing and solar panels, weights of the different subparts and also flying speed (Fig.4). Finally, depending on the application, a selection criterion will be defined. They can concern speed or wingspan, the UAV being stowed in a limited volume and launched by hand. With the help of the plot of Fig.4, a final configuration will be selected. In the case of the Solar plane project, a wingspan of $3.2 \mathrm{~m}$ and an aspect ratio of 13 were selected, which leads to a theoretical mass of $2.55 \mathrm{~kg}$. It is also interesting to plot the mass distribution in Fig.5, for example, to observe that the battery constitutes more than $40 \%$ of the entire weight. Between 2014 and 2015, a fully functional prototype was realized to validate this design methodology (Fig.6). The efficiencies and weight prediction models turned out to be very accurate, resulting in a total airplane mass of $2.506 \mathrm{~kg}$ and a total electrical power draw of $14 \mathrm{~W}$ for level flight, whereas the predicted power consumption was $14.2 \mathrm{~W}$. The wing is covered by a half square meter of silicon solar cells that offer a maximum power of $90 \mathrm{~W}$ at noon in summer. The prototype was 
tested successfully, using the sun as the only source of energy. This experiment proved the feasibility of continuous flight at constant altitude without using thermal soaring or storing potential energy by gaining altitude in the afternoon. It, however, requires extremely calm wind conditions, a good irradiance during the day, and especially no clouds at sunrise and sunset. Further information concerning this prototype can be found in.

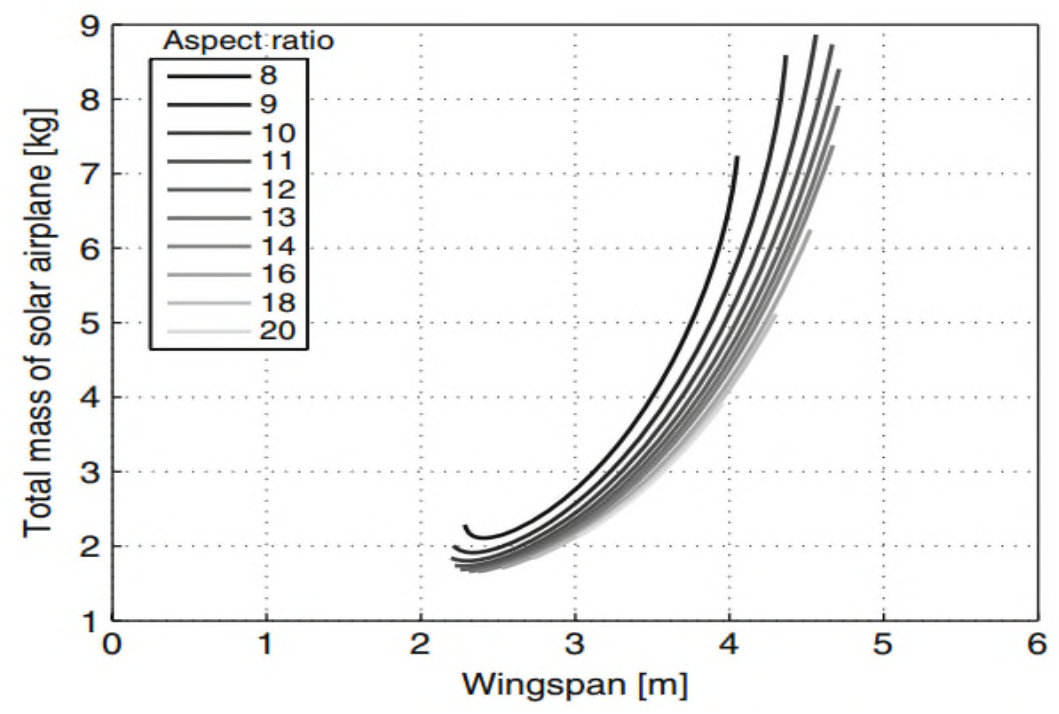

Fig. 3: Possible size configurations for a solar UAV embedding a 50g payload for a $24 \mathrm{~h}$ flight depending on the wingspan $\mathrm{b}$ and the aspect ratio AR.

\section{The Pros and Cons of Downscaling}

In the last section, we considered the application of our methodology and validated it at the UAV size, but now we can wonder how the feasibility of solar flight evolves with scaling. The analytical character of the design method and its mathematical models allows us to precisely discuss these scaling issues on the different airplane parts, which is the subject of the following subsections. 

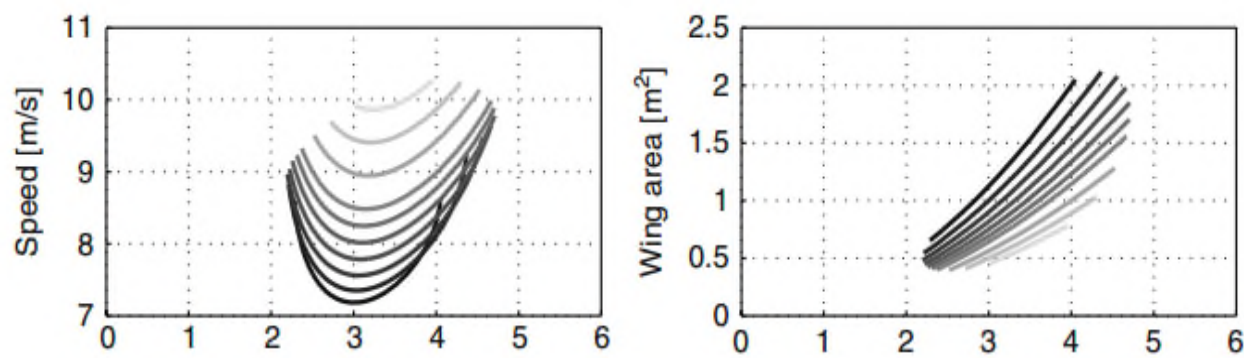

Aspect ratio
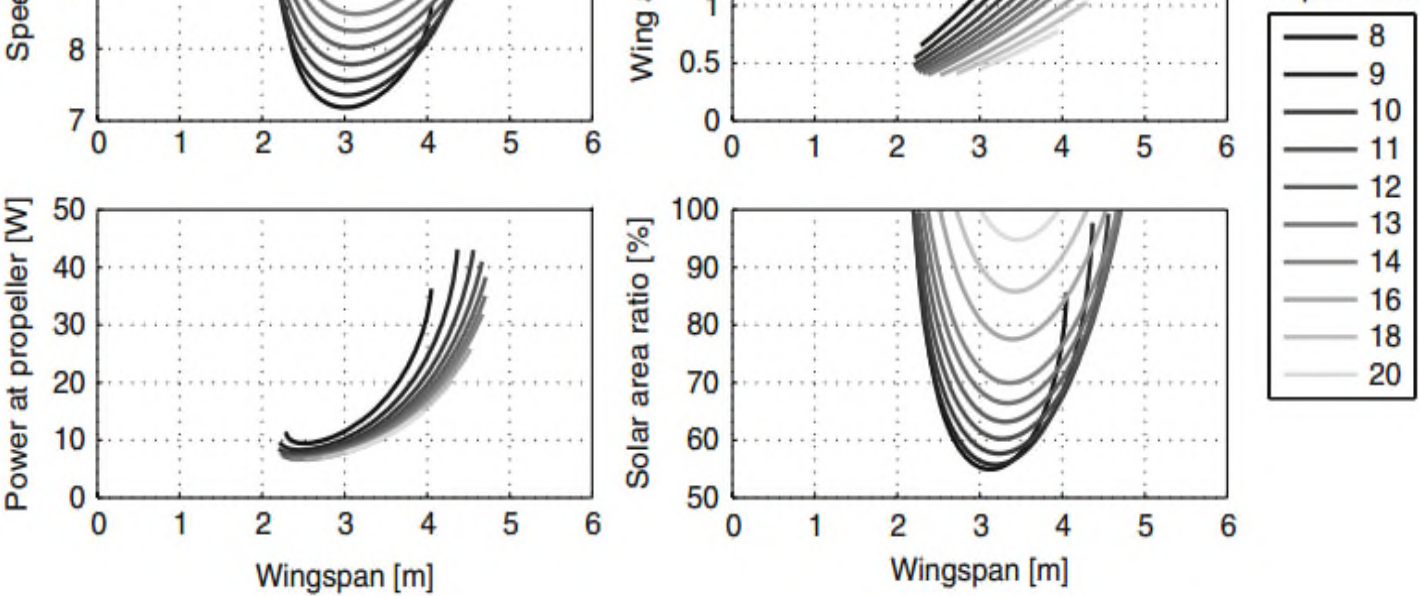

Fig. 4: Aircraft and flight characteristics depending on the wingspan $b$ and the aspect ratio $\mathrm{AR}$

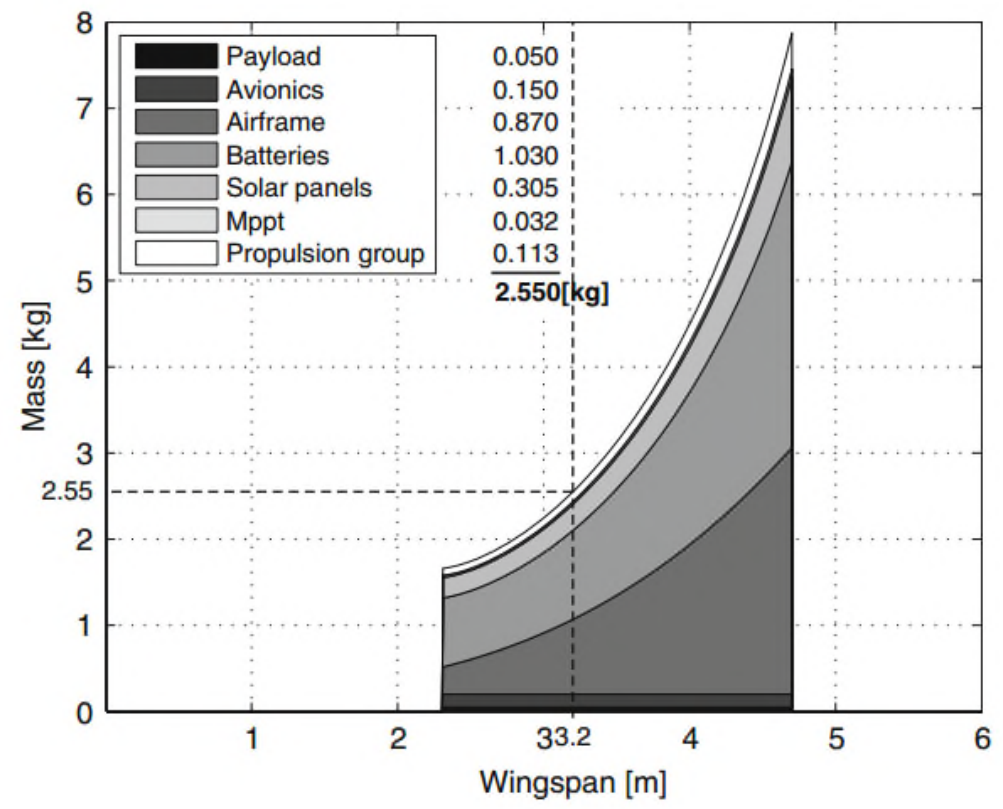

Fig. 5: Mass distribution for the solution with $A R=13$

\subsection{Airframe}

The airplane structure is the only part that scales down very well. In fact, its weight is proportional to the cube of a reference length, the wingspan for example. This property was highlighted by Tennekes in 1992 who presented, in his book "The simple 
science of flight" [4], very interesting correlations including insects, birds, and airplanes. He summarized the relations in a log-log diagram named "The Great Flight Diagram" where, following his own words, "everything that can fly" is represented. All the flying objects follow approximately a line with the following equation:

$$
\mathrm{W} / \mathrm{S}=47 \mathrm{~W}^{1 / 3}
$$

This cubic relation was also validated for sailplanes by the author with a database of $515 \mathrm{RC}$ models and manned sailplanes. A first interpolation was achieved to obtain the mean model after which only the best sailplanes, i.e., lighter than this mean model, were kept for a second interpolation. After five iterations of this process, only 5\% of the initial database remained to give the $5 \%$ best model, represented in Fig. 7 with the Great Flight Diagram. Our models are on the upper side of Tennekes curve which means that they are lighter, but at the same time more fragile. On the lower side, one can find the more robust flying systems, ranging from the house flies to combat aircrafts. These are heavier but able to withstand acrobatic maneuvers. The same figure also contains solar-powered airplanes flown to date. The available solar power relates linearly to the wing surface, thus square with the wingspan. The fact that the aircraft structure weight increases with the cube of the wingspan is a huge problem for large solar airplanes. As a matter of fact, it can be shown mathematically that the feasibility of these large solar airplanes implies a square and not a cubic relation between weight and wingspan. This ideal weight prediction model is represented with the gray region in Fig.7. It explains why existing large solar airplanes tend to get closer to these regions. At these large dimensions, this attempt to reduce mass leads to the construction of very fragile wings.

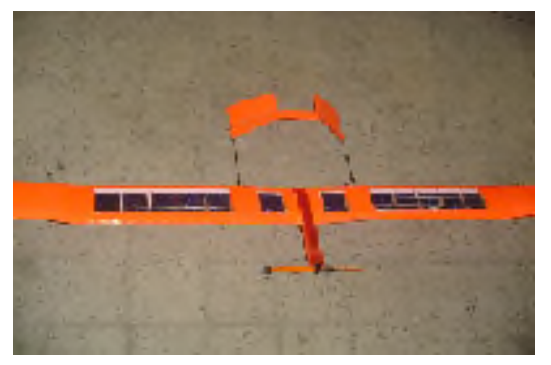

Fig. 6: The prototype of solar plane 


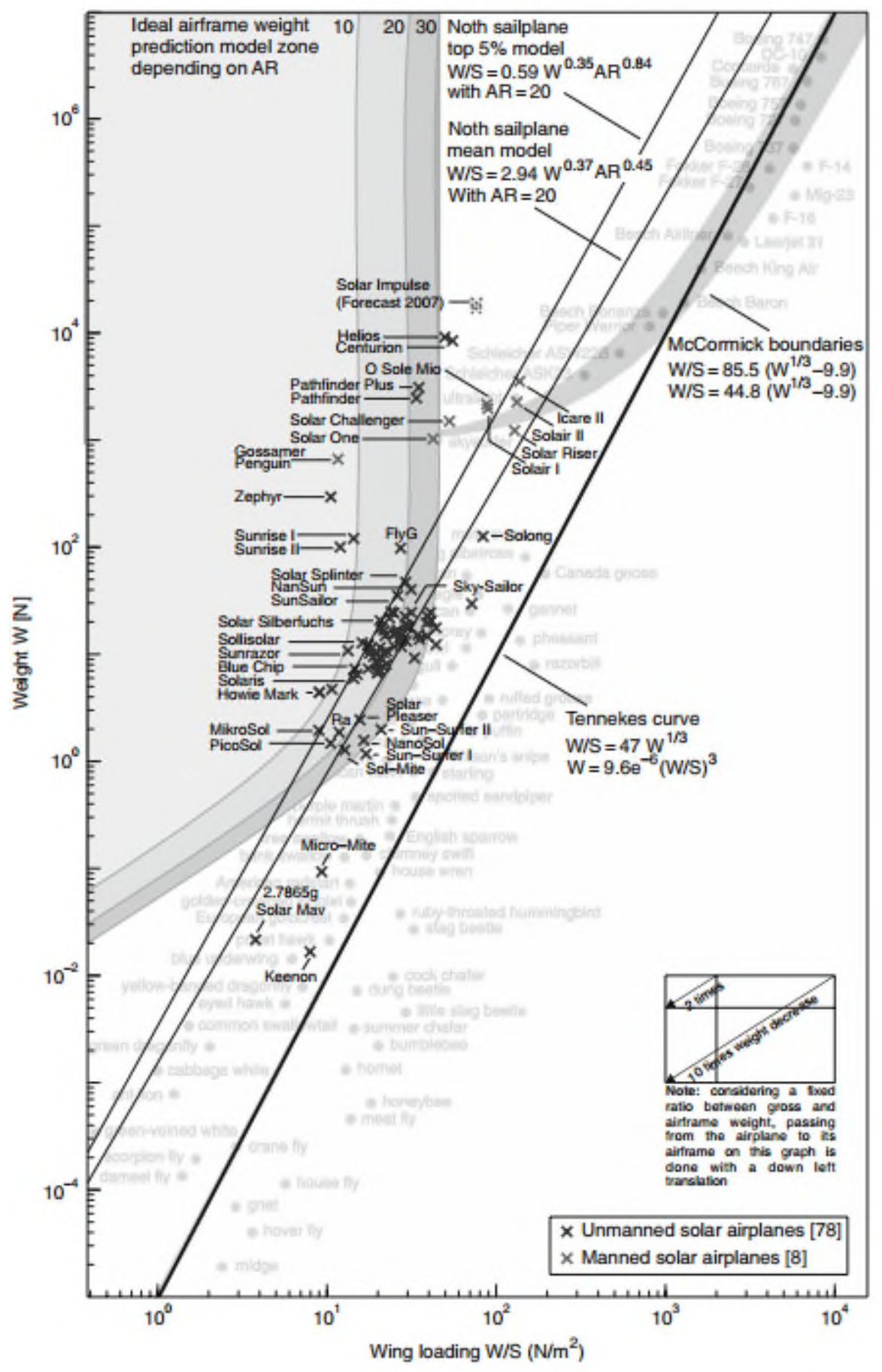

Fig. 7: The Flight Diagram

Law becomes a clear advantage. In fact, dividing the wingspan by a factor of 2 reduces the surface by 4 , but the weight of the structure by 8 . Additionally, the structure stiffness and the stress related to the mass scale linearly with the reference length. This is a great advantage for smaller systems which are intrinsically more robust against destruction forces related to their own mass. Also, an MAV has a much 
higher chance to survive a free fall than a big airplane because of the increasing ratio between air drag and mass. Nature gives us the example of ants that easily survive a fall from a multi-floor building whereas the elephant is seriously hurt when falling from around $1 \mathrm{~m}$.

\subsection{Low Reynolds Number Airfoil and Propeller}

Unfortunately, there are many drawbacks when scaling down an aircraft in size. On the aerodynamic side, it is well known that the lift to drag ratio decreases dramatically for Reynolds number smaller than $10^{5}$ (Fig.8).

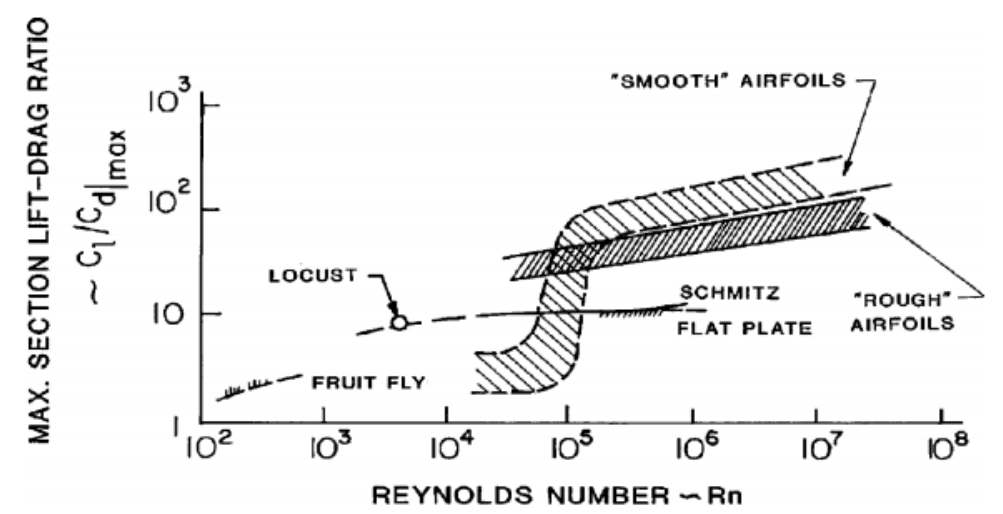

Fig. 8: Low Re number performance of smooth and rough airfoils.

\subsection{Actuators}

The downscaling of electro-magnetical motors is not favorable either. We have studied more than 20 motors, from $1 \mathrm{~mW}$ to $10 \mathrm{~kW}$, and it shows that the power to mass ratio is linear, hobbyist brushless motors being better than the industrial ones as demonstrated in Fig. 9. However, Fig.10 shows that the efficiency drops significantly below $1 \mathrm{~W}$. Here again, this is confirmed that has a motor-gearbox efficiency of $50 \%$. This leads to propulsion group efficiency $40 \%$. It emphasizes the fact that a very important point is the correct matching between motor, gearbox, and propeller. Unfortunately, the market offers far more products for the UAV than for the MAV range. It is thus even more difficult to find a good matching. The only remaining solution is to design and build a dedicated motor, gearbox, and propeller that fit 
perfectly to the application. For very low power, it seems that piezoelectric actuators could play an important role as their efficiency, poor compared to traditional electromagnetic motors at big dimensions, turns out to be more efficient than them at low dimensions. However, their command requires high voltages which induces more complex and heavier control electronics. Concerning the actuation of the control surfaces, servo motors are generally used for UAVs, but at the MAV size, it is more difficult to find lightweight and still reliable products. Alternative solutions are the use of magnet-in-a-coil actuators.

\subsection{Solar Cells}

Solar cells are also a problem, because they do not scale with the cube of the reference length but with the square. In fact, when reducing the wing surface of an MAV, we can put less cells but the thickness remains the same. Weight percentage then increases as compared to the total MAV mass. Another problem is that scaling down an airfoil decreases its curvature radius, making it far more difficult to install the fragile cells on a cambered wing. A solution to this problem is to place them flat inside the wing, closing the profile with a transparent sheet.

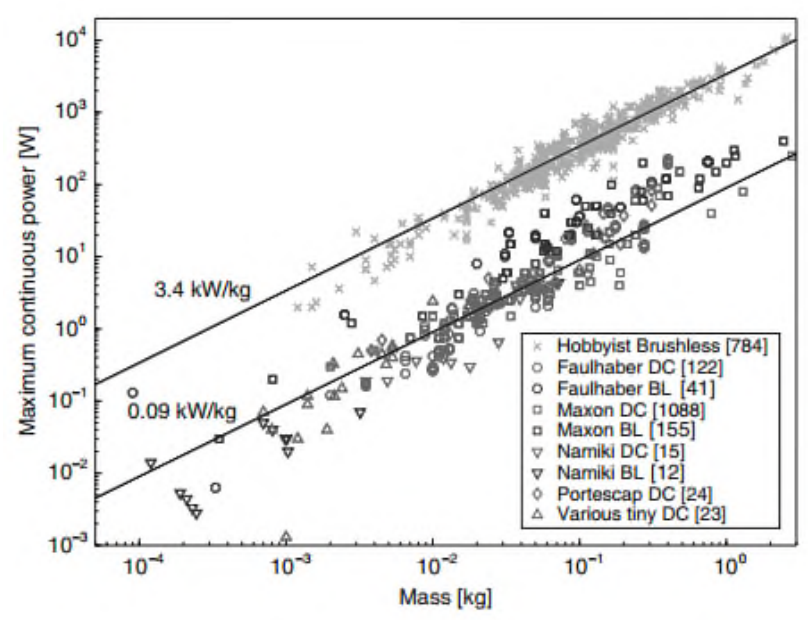

Fig. 9: Power to mass ratio for 2264 commercial motors from a database created with the specifications of various manufacturer's products. 


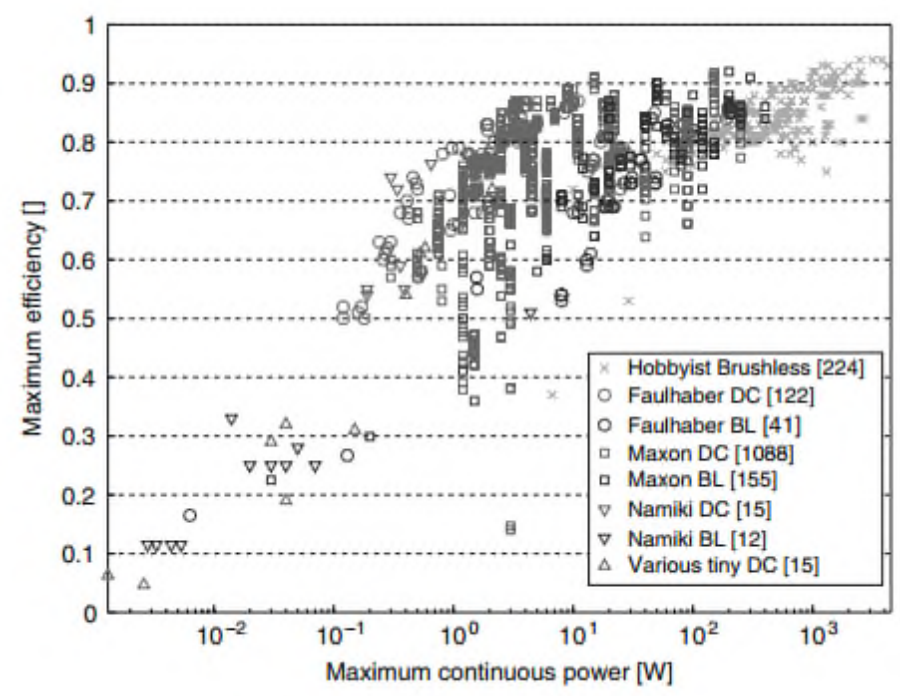

Fig. 10: Efficiency of 1672 motors vs maximum continuous power from a databasecreated with the specifications of various manufacturer's products.

This sheet must be non-reflective, otherwise it induces additional losses. Highly flexible solar cells (FlexCell, PowerFilm) can be applied on low curvature radius airfoils, but do not currently have high enough efficiency to be considered for continuous solar flight. They can, however, be used on an MAV that can recharge its batteries on the ground between two flights [1].

\subsection{Maximum Power Point Tracker}

The MPPT is responsible for interfacing the battery with the solar panels ensuring that they work at the maximum power point. Its efficiency also decreases at low dimensions where the operating voltage is reduced. This loss is due to the diode dropout voltage, which is $0.4 \mathrm{~V}$ for good Schottky diodes. In the case of the Solar plane that has a battery voltage of $30 \mathrm{~V}$, this is not critical, but on an MAV powered by a single lithium-ion cell of $3.7 \mathrm{~V}$, this translates into a loss of $11 \%$ of the source voltage. This is the reason why in some low-voltage designs, the solar cells are directly connected to the battery, choosing the number of solar cells in series so that their maximum power point voltage corresponds to the battery's nominal voltage. 
Bulletin de la Société Royale des Sciences de Liège, Vol. 85, 2016, p. 863 - 878

\subsection{Energy Storage}

On the side of energy storage, high gravimetric energy density lithium-ion or lithiumion-polymer cells are not easily scalable. Current battery technology is driven by the market of mobile devices; thus, the cells with the best specific energy are always of the 18650 type, a standard size in portable computer battery pack. In 2008, they offered energy densities of up to $240 \mathrm{Wh} / \mathrm{kg}$. They weigh at least $45 \mathrm{~g}$; thus, for a large airplane it is not problematic to use several of them, but they cannot be divided up for tiny MAVs. The only choice is then to select tiny batteries where the weight percentage of housing is higher, which inevitably reduces the energy density.

\subsection{Control}

If the MAV is aimed at being autonomous, the development of a navigation and control system becomes very critical at small scales, especially from the sensor side. It is no longer possible to embed GPS or IMU, the smallest of these two devices currently weighing around $10 \mathrm{~g}$ including the antenna for the GPS. Hence the expectations concerning the control capabilities have to be reduced. This limitation forces engineers to develop lightweight and low-power devices to sense the environment, taking inspiration from nature where optical flow is used to avoid walls. Also, the control of such MAVs itself is more difficult as they are more dynamic than larger UAVs. In fact, if we consider the angular acceleration formula $\mathrm{M}=\mathrm{I} \alpha$ and an airplane with a reference length 1 , the following reasoning can be done; moments on the airplane are the product of length and aerodynamic forces:

$\mathrm{M} \sim 1 \cdot \mathrm{F} \sim 1 \cdot \mathrm{S} \cdot \mathrm{v}^{2} \sim 1^{3} \cdot \mathrm{v}^{2}$

In order to find how the speed scales with the reference length, we can use the lift force and put it into equality with the weight, obtaining the flight speed at level flight $v=\sqrt{\frac{2 m g}{C_{L} \rho S}} \sim \sqrt{\frac{l^{3}}{l^{2}}} \sim \sqrt{l}$

Substituting Eq. (7) in Eq. (6) shows clearly that $M \sim 1^{4}$. Concerning the inertia, we know that

$\mathrm{I} \sim \mathrm{m} \cdot \mathrm{1}^{2} \sim 1^{5}$

Considering again the angular acceleration formula, we can write 


$$
\alpha=\frac{M}{I} \sim \frac{l^{4}}{l^{5}} \sim \frac{1}{l}
$$

This result proves that the smaller an aerial vehicle is, the more dynamic it is. Consequently in the case of MAVs, it tends to be more difficult to maintain a constant angle of attack and thus stay at an optimal angle where an aerodynamic characteristic like the lift to drag ratio is the highest.

\section{Conclusion}

The development of solar airplanes has seen the realization of many prototypes bigger than a meter, but investigations into their feasibility below this limit are very rare. In this chapter, a very simple methodology was developed for the design of this particular type of flying platform, focusing particularly on models with a wingspan below $1 \mathrm{~m}$. Unfortunately, downscaling introduces many problems which makes the realization of an efficient solar only MAV impossible at this time. Existing models use a battery coupled to solar cells that only increase the flight time. The main limitations are the low efficiencies of the propulsion group and the deterioration of the aerodynamics characteristics, due to low Reynolds numbers. Additionally, the choice of components, i.e., motor, propellers, batteries, etc., on the market is very poor at this size so it turns out that the realization of a solar MAV first requires a careful design and the manufacturing of dedicated parts specifically for the application. Finally, the design of solar-powered MAVs also requires major improvements on the side of flexible and efficient solar cells, small and high energy density batteries, and lightweight sensors and actuators. For many of these issues that prevent us to build an MAV with a size of a few centimeters and a reasonable autonomy, a quick look at insects shows us that biology is still far ahead from science. One flagrant example that illustrates this fact is energy storage, where sugar has 20 times more specific energy than the best currently available lithium-ion technology. It will thus require several more decades to be able to compete with nature. 
Bulletin de la Société Royale des Sciences de Liège, Vol. 85, 2016, p. 863 - 878

\section{Acknowledgments}

The author would like to thank Dr. Mohammadhoseyn Goli for the outstanding contributions on the realization of the Rad-Solar plane prototype.

\section{References}

1. Fuchs, J.P.: Solar Cell System for the Microglider. Bachelor Thesis, Laboratory of Intelligent Systems, EPFL, Lausanne (2008)

2. McCormick, B.W.: Aerodynamics, Aeronautics, and Flight Mechanics. John Wiley, New-York (1995)

3. Pines, D.J., Bohorquez, F.: Challenges Facing Future MicroAir-Vehicle Development. Journal of Aircraft 43(2), 290- 305 (2006)

4. Tennekes, H.: The Simple Science of Flight - From Insects to Jumbo Jets. MIT Press, Cambridge, Massachusetts, USA (1992) 